\title{
Laser Induced Breakdown Spectroscopy of Er II for Transition Probability Measurements
}

\author{
Yuki Naoi ${ }^{1, \dagger}$, Masayuki Iwata ${ }^{1, \dagger}{ }^{\text {, Daichi Yokota }}{ }^{1, \dagger}$, Gediminas Gaigalas ${ }^{2}$ (D) Daiji Kato ${ }^{3,4}$, Izumi Murakami ${ }^{3,5}$ ID , \\ Hiroyuki A. Sakaue ${ }^{3}$, Yuichiro Sekiguchi ${ }^{6}$, Masaomi Tanaka ${ }^{7}$, Hajime Tanuma ${ }^{8}$, Shinya Wanajo ${ }^{9,10}$ \\ and Nobuyuki Nakamura 1,3,*(D)
}

1 Institute for Laser Science, The University of Electro-Communications, Tokyo 182-8585, Japan; m_iwata@ils.uec.ac.jp (M.I.)

2 Institute of Theoretical Physics and Astronomy, Vilnius University, 10257 Vilnius, Lithuania; Gediminas.Gaigalas@tfai.vu.lt

3 National Institute for Fusion Science, Gifu 509-5292, Japan; kato.daiji@nifs.ac.jp (D.K.); murakami.izumi@nifs.ac.jp (I.M.); sakaue.hiroyuki@nifs.ac.jp (H.A.S.)

4 Interdisciplinary Graduate School of Engineering Sciences, Kyushu University, Fukuoka 816-8580, Japan

5 Department of Fusion Science, The Graduate University for Advanced Studies, SOKENDAI, Gifu 509-5292, Japan

6 Department of Physics, Toho University, Chiba 274-8510, Japan; y.sekiguchi@sci.toho-u.ac.jp

7 Astronomical Institute, Tohoku University, Sendai 980-8578, Japan; masaomi.tanaka@astr.tohoku.ac.jp

8 Department of Physics, Tokyo Metropolitan University, Tokyo 192-0397, Japan; tanuma-hajime@tmu.ac.jp

9 Max-Planck-Institut für Gravitationsphysik (Albert-Einstein-Institut), 14476 Potsdam, Germany; shinya.wanajo@aei.mpg.de

10 Interdisciplinary Theoretical and Mathematical Sciences Program (iTHEMS), RIKEN, Saitama 351-0198, Japan

* Correspondence: n_nakamu@ils.uec.ac.jp

+ These authors contributed equally to this work.

check for updates

Citation: Naoi, Y.; Iwata, M.; Yokota, D.; Gaigalas, G.; Kato, D.; Murakami, I.; Sakaue, H.A.; Sekiguchi, Y.; Tanaka, M.; Tanuma, H.; et al. Laser Induced Breakdown Spectroscopy of Er II for Transition Probability Measurements. Appl. Sci. 2022, 12, 2219. https://doi.org/10.3390/ app12042219

Academic Editor: Agresti Juri

Received: 11 January 2022

Accepted: 10 February 2022

Published: 21 February 2022

Publisher's Note: MDPI stays neutral with regard to jurisdictional claims in published maps and institutional affiliations.

Copyright: () 2022 by the authors. Licensee MDPI, Basel, Switzerland. This article is an open access article distributed under the terms and conditions of the Creative Commons Attribution (CC BY) license (https:// creativecommons.org/licenses/by/ $4.0 /)$.
Abstract: We present a laser induced breakdown spectrum of Er II in the near ultraviolet region. To use the spectrum for the evaluation of the transition probabilities, an alloy target with a low content of Er was used to suppress the self-absorption. From the linearity of the Boltzmann plot obtained by using the sensitivity corrected experimental intensity and existing transition probability data, the local thermal equilibrium condition of the plasma and the reliability of the transition probability data are confirmed. The linear function obtained in the Boltzmann plot is used for the determination of a previously unreported transition probability for the line at $393.863 \mathrm{~nm}$.

Keywords: laser induced breakdown spectroscopy; transition probabilities; lanthanides; erbium; neutron star merger

\section{Introduction}

Spectroscopic data of rare-earth elements are important for several fields, especially in astrophysics. For example, data of transition wavelength and probability are essential to estimate the amount of these elements in the stellar atmosphere [1,2]. In addition, such atomic data sets are needed for the accurate radiative transfer simulation for kilonovae produced by neutron star mergers [3,4]. Er is one of the most important among rare-earth elements.

In the NIST atomic spectra database (ASD) [5], 285 lines of Er II are registered on the basis of the data compilation by Meggers et al. [6] in 1975. Among them, only 11 lines are listed with energy level classifications and transition probabilities. Musioł and Łabuz [7] measured relative transition probabilities of 101 lines by arc plasma spectroscopy. By normalizing the relative data to the experimental radiative lifetime by Bentzen and Nielsen [8], absolute values were determined. Xu et al. [9] measured radiative lifetimes of 30 excited levels by time-resolved laser-induced fluorescence (LIF). By using theoretical branching fractions, transition probabilities of 32 lines were obtained. 
Significant progress was made by Lawler et al. [10] (this paper is referred to as Lawler2008 hereafter), who determined the transition probabilities of 418 lines from their branching fraction measurements with a combination of radiative lifetimes measured by Stockett et al. [11]. Some of the transition probability data in Lawler2008 were evaluated in a recent experimental study by Yu et al. [12] using a time resolved laser induced fluorescence method. Good agreement in the $g A$ values found between their data implies the reliability of the transition probabilities in Lawler2008. Wyart and Lawler [13] extended the study with the aid of theoretical analysis, using the Cowan code [14], and 130 levels of even parity and 230 levels of odd parity were summarized with configuration mixing coefficients. Ankush and Deo [15] estimated the isotope shift between ${ }^{166} \mathrm{Er}$ and ${ }^{170} \mathrm{Er}$, using the mixing coefficients derived by Wyart and Lawler, and found that the estimated shifts were consistent with the experimentally measured shifts.

In this paper, we present an emission spectrum of Er II, obtained by laser induced breakdown spectroscopy (LIBS), where the emission from plasma produced by an intense laser pulse is observed. LIBS has been mainly developed for element analysis in industrial manufacturing [16], environmental monitoring [17,18], geological studies [19] including planetary exploration [20], and many other applications. On the other hand, LIBS is one of the most powerful methods for the determination of fundamental atomic properties, such as transition probabilities (see the review paper [21] in this Special Issue and references therein). In order to determine transition probabilities properly, a local thermal equilibrium (LTE) condition should be realized. Although the temperature of the plasma varies with time, instantaneous observation of the plasma with a time-resolved intensified camera can ensure the LTE condition. Since the emission intensity $I$ in the LTE condition is proportional to the product of the transition probability $A$ and the Maxwell-Boltzmann population determined by the temperature $T$ and the upper level energy $E$, the logarithm of $I / g A$, where $g$ is the statistical weight, should have a linear dependence on $E$ (see Section 2). The linearity can be used to confirm the reliability of the $A$ values used. Once the linearity is confirmed, unknown $A$ values can be obtained by applying the experimental intensity to the linear function obtained. LIBS is a simple method for evaluating or accumulating transition probability data, compared with other methods such as LIF. It also has the advantage that many transitions can be evaluated at the same time and that metal targets can be used as is, without vaporization. From the LIBS spectrum, we confirm the linearity by using the $A$ values in Lawler2008 and a previously unreported transition probability is derived from the obtained linearity.

\section{Experiment}

Figure 1 shows a schematic of the experimental setup. A Q-switched Nd:YAG laser (Spectra Physics GCR-150) was used in the fundamental mode $(1064 \mathrm{~nm})$ for producing plasma. Laser pulses with a width of 8-9 ns and an energy of about $15 \mathrm{~mJ}$ were focused with a $150 \mathrm{~mm}$ focal length lens and introduced through a fused silica window. The pulse repetition frequency was $10 \mathrm{~Hz}$.

For the determination of transition probabilities, it is important to realize a thin plasma condition [21] as well as the LTE condition. If the plasma is so thick that it can absorb the emission occurring within the plasma (self-absorption effect), the observed intensity does not reflect the transition probability. An $\mathrm{Al}$ alloy with a low content $(5 \mathrm{wt} \% \sim 0.8$ at $\%$ ) of Er was thus used as a target in the present experiment for producing thin Er plasma. A rod-shaped target with a $6 \mathrm{~mm}$ diameter was installed in the chamber. The target rod was rotated while being moved up and down with a motion feedthrough system similar to that used in [22]. The target motion enabled each laser pulse to hit the unirradiated surface of the target. The target chamber was filled with $200 \mathrm{~Pa}$ of Ar after evacuating the chamber with a scroll pump. 


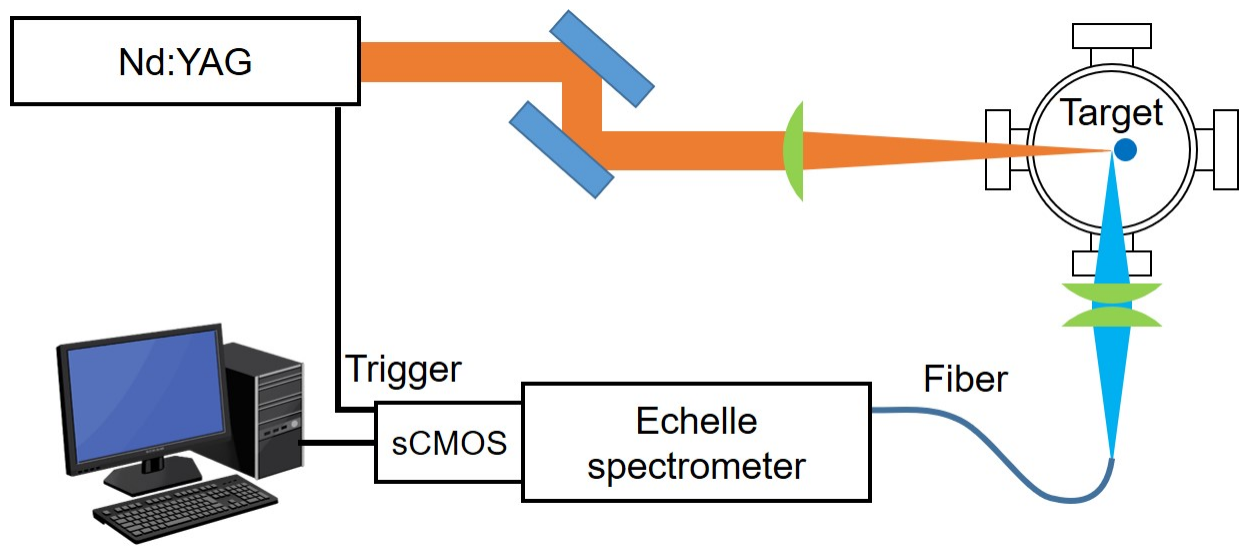

Figure 1. Schematic diagram of the experimental setup.

The emission from the plasma was observed through a fused silica window placed at $90^{\circ}$ with respect to the laser direction. A set of synthetic silica plano-convex lenses was used to focus the emission onto the entrance of the fiber connected to an echelle spectrometer (LTB ARYELLE 200). A fast-gated image-intensified sCMOS (Andor iStar-sCMOS-18F-03) was used as a detector. The spectral resolution (specification value) is 9200 at $253 \mathrm{~nm}$ and 8000 at $404 \mathrm{~nm}$. The data acquisition period was $1 \mu \mathrm{s}$ after a delay of $5 \mu \mathrm{s}$ from each laser pulse. The timing was controlled by a digital delay generator installed in the sCMOS detector. The spectrometer response function was obtained using a calibration light source (Ocean Optics DH-3plus-BAL-CAL).

The emission line intensity $I$ (photon number emitted in a unit of time) is determined by the product of the population of the upper level and the transition probability $A$. It is thus represented as the following equation for the lines emitted from the local thermal equilibrium (LTE) plasma with a temperature $T$ :

$$
I=g A \cdot C \exp \left(-\frac{E}{k T}\right),
$$

where $g, E$, and $k$ represent the statistical weight, the energy of the upper level, and the Boltzmann constant, respectively, and $C$ is a constant depending on the ion species. By taking the natural logarithm, we obtain

$$
\ln \left(\frac{I}{g A}\right)=-\frac{E}{k T}+C^{\prime},
$$

where $C^{\prime}$ is a new constant. Thus the plot of the left side value as a function of $E$ (Boltzmann plot) should indicate linearity when the LTE condition is established.

\section{Results and Discussion}

Figure 2a shows the LIBS spectrum for the whole spectrometer region $(200-1080 \mathrm{~nm})$ obtained by about 1000 shot accumulations. Close-up views of the 300-400 nm, 330-340 nm and $390-400 \mathrm{~nm}$ regions are also shown in Figure $2 \mathrm{~b}-\mathrm{d}$, respectively. In addition to the emission lines of Er, lines from $\mathrm{Ar}$ (atmospheric gas) and $\mathrm{Al}$ (the main content of the target alloy) were also confirmed, including strong lines of $\mathrm{Al} \mathrm{I} \mathrm{[5]} \mathrm{at} \mathrm{around} 310 \mathrm{~nm}$ and $395 \mathrm{~nm}$. Among the observed Er lines, $30 \mathrm{Er}$ II lines in Lawler2008 were found to have enough intensity to analyze. All of them, which are listed in Table 1, were concentrated in the $300 \mathrm{~nm}$ range shown in Figure 2b. For example, in the $330-340 \mathrm{~nm}$ range shown in Figure 2c, eight lines from Er II were observed, as indicated by the red arrows. They are well represented by a Gaussian line shape, as shown in the inset for the $337.271 \mathrm{~nm}$ line as an example. The peak area of each observed line was thus obtained by fitting a Gaussian function, and the relative intensity I was obtained, as listed in Table 1, by correcting the peak area with the response function obtained with the calibration light source. 


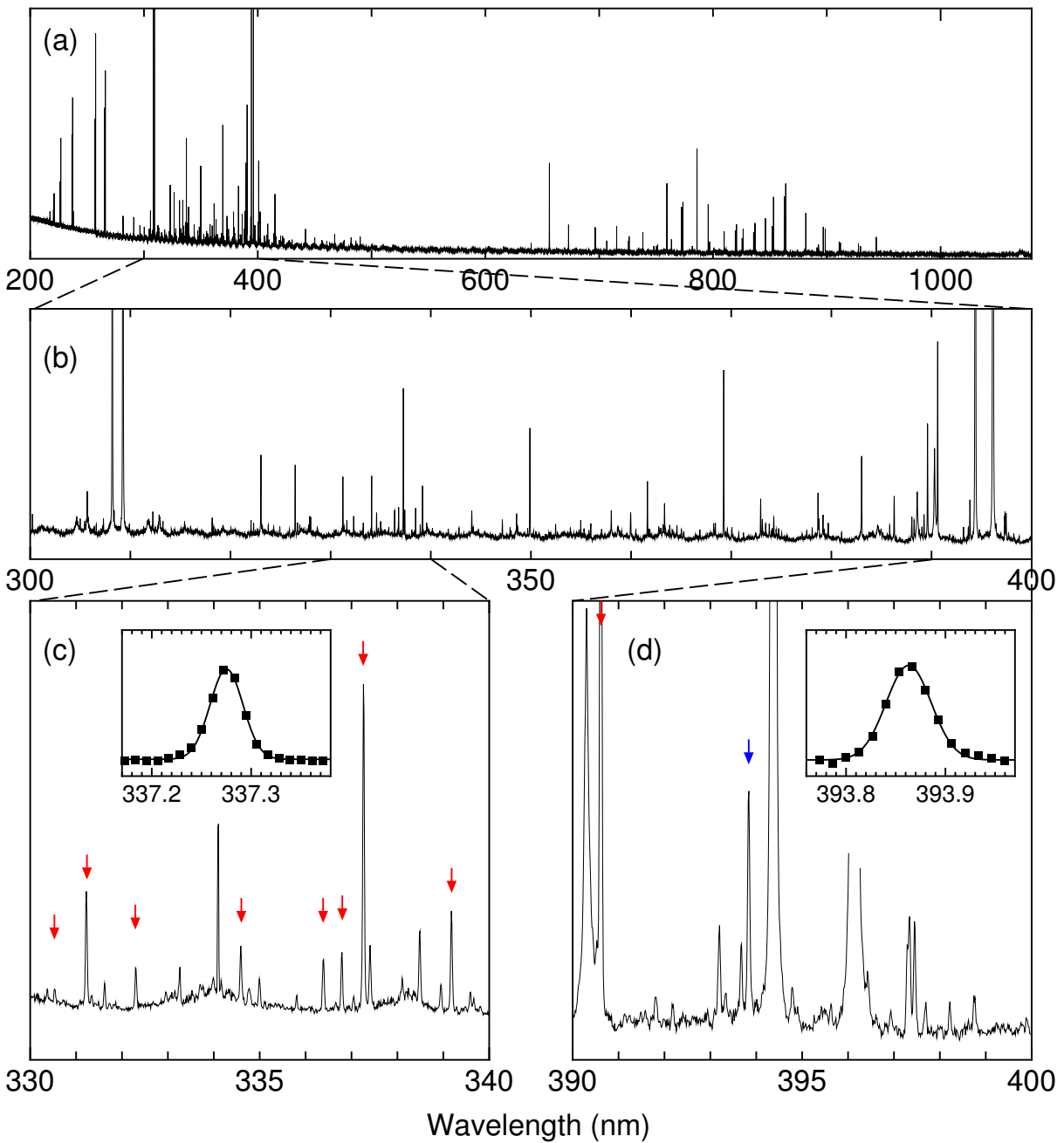

Figure 2. LIBS spectrum obtained for an Al-5 wt $\%$ Er alloy target in an Ar atmosphere for the (a) whole spectrometer (200-1080 nm), (b) 300-400 nm, (c) 330-340 nm, and (d) 390-400 nm regions. It is noted that the sensitivity correction is not applied. It is also noted that the undulated baseline of the spectrum reflects the response of each diffraction order of the Echelle grating. The lines indicated by the red arrows represent those given in Lawler2008 [10]. The blue arrow indicates the $393.863 \mathrm{~nm}$ line listed in the NIST database without a transition probability.

The self-absorption effect can be estimated from the line intensity ratio between the intense transitions with the same upper level $[23,24]$. When the thin condition is valid, the intensity ratio of such a transition pair should be equivalent to the branching ratio determined only by the transition probability ratio. On the other hand, when the thin condition is not valid, the intensity ratio of such a pair tends to deviate from the branching ratio, as the self-absorption effect decreases the observed intensity more significantly for the transition with a larger transition probability. In the present spectrum, the ratio between the $389.623 \mathrm{~nm}$ and the $383.048 \mathrm{~nm}$ lines, which are relatively intense and have the same upper level with an energy of $3.236 \mathrm{eV}$, is a suitable probe for the self-absorption effect. The sensitivity corrected experimental intensity ratio for those lines was $1.2 \pm 0.2$, which is in good agreement with the branching ratio $1.23 \pm 0.09$ [10] within the uncertainties. We also measured this ratio using a target with twice the content (10 $\mathrm{wt} \% \sim 1.8$ at $\%$ ) of Er. Although consistency with the branching ratio was also confirmed for the higher content target, we used the lower content ( $5 \mathrm{wt} \% \sim 0.8$ at $\%$ ) target to ensure the thin plasma condition more certainly. 
Table 1. Transitions used in the present analysis. Wavelength $(\lambda)$, parity, $2 J$ value, level energy $(E)$, and transition probability $(A)$ are taken from Lawler2008 [10] except for the $393.863 \mathrm{~nm}$ line. For the $393.863 \mathrm{~nm}$ line, wavelength, parity, $2 J$ value, and level energy taken from the NIST database [5] and the transition probability obtained in the present measurement are listed. The sensitivity-corrected relative intensities $I$ in the present LIBS spectrum, normalized to the $390.631 \mathrm{~nm}$ line, are listed for all the lines.

\begin{tabular}{|c|c|c|c|c|c|c|c|c|}
\hline \multirow[b]{2}{*}{$\lambda(\mathrm{nm})$} & \multicolumn{3}{|c|}{ Lower } & \multicolumn{3}{|c|}{ Upper } & \multirow{2}{*}{$\begin{array}{c}A \\
\left(10^{6} s^{-1}\right)\end{array}$} & \multirow[b]{2}{*}{$I$} \\
\hline & Parity & $2 J$ & $E(\mathrm{eV})$ & Parity & $2 J$ & $E(\mathrm{eV})$ & & \\
\hline 290.447 & odd & 15 & 0.846164 & even & 17 & 5.113654 & $93 \pm 5$ & 0.05 (1) \\
\hline 291.036 & odd & 15 & 0.846164 & even & 15 & 5.105013 & $204 \pm 11$ & 0.12 (1) \\
\hline 296.452 & odd & 15 & 0.846164 & even & 17 & 5.027212 & $159 \pm 8$ & $0.14(2)$ \\
\hline 300.241 & odd & 15 & 0.846164 & even & 13 & 4.974455 & $130 \pm 7$ & $0.14(2)$ \\
\hline 323.058 & even & 11 & 0.054607 & odd & 11 & 3.891327 & $92 \pm 5$ & $0.42(5)$ \\
\hline 323.798 & even & 9 & 0.636362 & odd & 9 & 4.464322 & $19.1 \pm 1$ & $0.05(1)$ \\
\hline 326.478 & even & 13 & 0.000000 & odd & 13 & 3.796533 & $69 \pm 3$ & $0.44(5)$ \\
\hline 330.557 & even & 9 & 0.892110 & odd & 9 & 4.641802 & $32.1 \pm 1.7$ & $0.03(1)$ \\
\hline 331.243 & even & 11 & 0.054607 & odd & 13 & 3.796533 & $40.9 \pm 2.1$ & $0.31(3)$ \\
\hline 332.319 & even & 9 & 0.636362 & odd & 11 & 4.366164 & $34.3 \pm 1.9$ & $0.11(1)$ \\
\hline 334.603 & even & 11 & 0.054607 & odd & 9 & 3.758950 & $25.6 \pm 1.3$ & $0.15(2)$ \\
\hline 336.408 & even & 11 & 0.054607 & odd & 11 & 3.739083 & $18.5 \pm 0.9$ & $0.17(2)$ \\
\hline 336.802 & even & 11 & 0.054607 & odd & 11 & 3.734768 & $18.8 \pm 0.9$ & $0.16(2)$ \\
\hline 337.275 & even & 13 & 0.000000 & odd & 15 & 3.674999 & $145 \pm 7$ & $0.89(10)$ \\
\hline 339.199 & even & 13 & 0.000000 & odd & 15 & 3.654160 & $28.3 \pm 1.4$ & 0.29 (3) \\
\hline 349.910 & even & 11 & 0.054607 & odd & 9 & 3.596907 & $105 \pm 5$ & $0.62(7)$ \\
\hline 359.950 & odd & 15 & 1.402160 & even & 13 & 4.845660 & $52.2 \pm 2.7$ & $0.20(2)$ \\
\hline 360.490 & odd & 19 & 1.588866 & even & 17 & 5.027212 & $42 \pm 3$ & $0.07(1)$ \\
\hline 361.657 & even & 13 & 0.000000 & odd & 11 & 3.427253 & $21 \pm 1.1$ & $0.37(4)$ \\
\hline 369.265 & even & 11 & 0.054607 & odd & 13 & 3.411246 & $67 \pm 3$ & $0.95(10)$ \\
\hline 370.764 & odd & 15 & 1.770584 & even & 17 & 5.113654 & $46 \pm 4$ & $0.06(1)$ \\
\hline 373.127 & even & 9 & 0.892110 & odd & 11 & 4.214012 & $19.5 \pm 1$ & $0.08(1)$ \\
\hline 373.816 & odd & 13 & 1.619322 & even & 15 & 4.935095 & $46.8 \pm 2.5$ & $0.06(1)$ \\
\hline 374.264 & even & 9 & 0.636362 & odd & 9 & 3.948168 & $20.6 \pm 1.1$ & $0.11(1)$ \\
\hline 383.048 & even & 13 & 0.000000 & odd & 13 & 3.235860 & $19.4 \pm 1$ & $0.49(6)$ \\
\hline 385.839 & even & 9 & 0.892110 & odd & 11 & 4.104563 & $18.5 \pm 0.9$ & $0.08(1)$ \\
\hline 388.061 & even & 9 & 0.636362 & odd & 7 & 3.830423 & $31.3 \pm 1.6$ & $0.15(2)$ \\
\hline 388.289 & even & 11 & 0.886441 & odd & 9 & 4.078630 & $31.6 \pm 1.6$ & $0.12(2)$ \\
\hline 389.623 & even & 11 & 0.054607 & odd & 13 & 3.235860 & $23.9 \pm 1.2$ & $0.59(6)$ \\
\hline 390.631 & even & 13 & 0.000000 & odd & 11 & 3.173046 & $48.2 \pm 2.4$ & $1.00(10)$ \\
\hline 393.863 & even & 13 & 0.000000 & odd & 11 & 3.147014 & $12 \pm 2$ & $0.26(3)$ \\
\hline
\end{tabular}

The left side values of Equation (2) obtained from the experimental intensity $I$ and the $g A$ values in Lawler2008 are plotted in Figure 3 as a function of the energy $E$ of the upper level of the transition. The error bars represent the uncertainty obtained considering four contributions: (i) the fitting error, (ii) the statistical uncertainty of the photon counts estimated from the conversion factor from the digital counts to the photon number, (iii) the uncertainty in the radiometric data of the calibration light source, and (iv) the uncertainty in the $A$ values. The linear function corresponding to Equation (2) was obtained by weighted fitting to the data, as shown by the solid line. The $68.3 \%$ confidence interval is also shown by the dashed curves. From the gradient of the fitted linear function, the plasma temperature is derived to be $6700 \pm 400 \mathrm{~K}$.

As seen in the figure, the Boltzmann plot shows rather a good linearity although some of the data are scattered from the fitted linear function. In general, the linearity in the Boltzmann plot ensures the LTE condition of the plasma and the reliability of the $g A$ values. In other words, the deviation of the data from the linear dependence should be attributed to the deviation from the LTE condition or the error in the $g A$ value (or both). We consider that the scattering is due to an incomplete LTE condition in the present experiment, as the reliability of the data in Lawler2008 was confirmed through comparison with the transition 
probability data independently obtained by a different method from another group [12], as described in Introduction. However, the large deviation for the data at $E=4.85 \mathrm{eV}$ may imply an underestimation of the $g A$ value for the corresponding transition $(359.950 \mathrm{~nm})$. If the data is applied to the obtained linear function, an $A$ value of $1.5(2) \times 10^{8} \mathrm{~s}^{-1}$ is obtained, whereas it is $5.22(27) \times 10^{7} \mathrm{~s}^{-1}$ in Lawler2008.

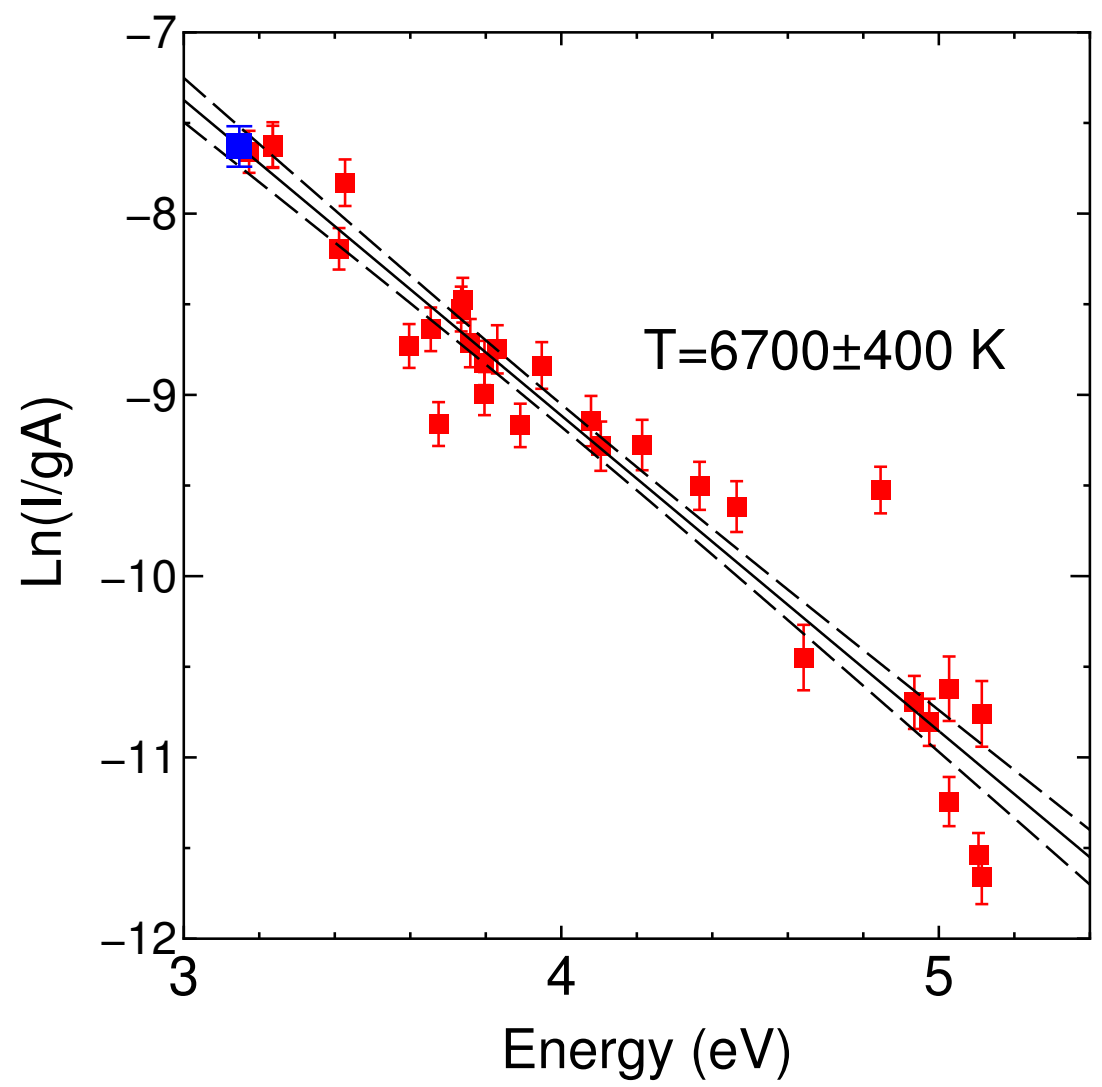

Figure 3. Boltzmann plot obtained using the present experimental line intensity $I$ and the $g A$ values taken from Lawler2008 [10] (red squares). The fitted linear function and the $68.3 \%$ confidential interval are shown in the solid and dashed lines, respectively. The blue square represents the linear function value at $3.147 \mathrm{eV}$ for obtaining the $A$ value of the $393.863 \mathrm{~nm}$ line (see text for details).

In the NIST database, there is one transition at $393.863 \mathrm{~nm}$ for which the lower and upper level energies are given but the transition probability is not given. The transition probability of this line is not included either in Lawler2008. As listed in the last line in Table 1, the angular momentum and the parity of the upper level are defined as $J=11 / 2$ and odd in the NIST database, but the electron configuration is not given. According to Wyart and Lawler [13], $4 f^{11} 5 d 6 s$ is assigned to the corresponding level although $4 f^{11} 6 s^{2}, 4 f^{12} 6 p$, and $4 f^{11} 5 d^{2}$ are mixed with a mixing coefficient of $0.37,12.55$, and $2.71 \%$, respectively.

This $393.863 \mathrm{~nm}$ line was observed in the present LIBS spectrum, as indicated by the blue arrow in Figure 2d, and the intensity was obtained from the peak area of the fitted Gaussian function shown in the inset. By applying this line to the linear function in Figure 3, as plotted by the blue square, the transition probability $A$ is obtained as $1.2(2) \times 10^{7} \mathrm{~s}^{-1}$. The uncertainty was estimated from three contributions: (i) uncertainty in the experimental intensity, (ii) uncertainty in radiometric data of the calibration light source, and (iii) the confidence interval of the linear function obtained from the Boltzmann plot, among which the third contribution is dominant.

Our work demonstrated that measurements of the LIBS spectra provide an effective way to confirm the reliability of the existing transition probability data. In addition, we showed that, when there are some existing data of transition probabilities for a certain ion, the LIBS spectra give an estimation of unknown transition probabilities for the lines 
with identified upper levels. In particular, the latter aspect is useful to systematically give transition probabilities of the lanthanide elements, for which extensive datasets are not available.

\section{Summary}

In the present laser induced breakdown spectroscopy experiment, emission from $\mathrm{Er}$ II excited in the plasma produced by a Q-switched Nd:YAG laser was observed, using an echelle spectrometer with a fast gated intensified detector. The local thermal equilibrium condition of the plasma was confirmed by the linearity in the Boltzmann plot, obtained from the fast-gated spectrum and existing reliable transition probability data. The transition probability of a line at $393.863 \mathrm{~nm}$, which has previously not been reported, was determined by applying its intensity to the linear function obtained in the Boltzmann plot to be $1.2(2) \times 10^{7} \mathrm{~s}^{-1}$.

Author Contributions: Conceptualization, G.G., D.K., I.M., Y.S., M.T., H.T., S.W. and N.N.; investigation, Y.N., M.I., D.Y. and N.N.; writing—original draft preparation, N.N.; writing—review and editing, D.K., M.T., H.T. and S.W.; funding acquisition, D.K., H.A.S., M.T. and N.N. All authors have read and agreed to the published version of the manuscript.

Funding: This work was supported by JSPS KAKENHI Grant Number JP19H00694, the NIFS Collaboration Research Program (NIFS21KOAF006), the NINS program for cross-disciplinary science study, the NINS program of Promoting Research by Networking among Institutions (Grant Number 01411702), the Japan Society for the Promotion of Science (JSPS) the Bilateral Joint Research Project, and JSPS KAKENHI Grant Number JP18H01201.

Institutional Review Board Statement: Not applicable.

Informed Consent Statement: Not applicable.

Data Availability Statement: The data presented in this study are available from the corresponding author on request.

Acknowledgments: N.N. would like to thank M. G. Su and D. X. Sun at Northwest Normal University for providing us with technical information on LIBS.

Conflicts of Interest: The authors declare no conflict of interest.
Abbreviations
The following abbreviations are used in this manuscript:
LIBS Laser induced breakdown spectroscopy
NIST National Institute of Standards and Technology
LIF Laser induced fluorescence
LTE Local thermal equilibrium
CMOS Complementary metal oxide semiconductor

\section{References}

1. Sneden, C.; Cowan, J.J.; Gallino, R. Neutron-Capture Elements in the Early Galaxy. Annu. Rev. Astron. Astrophys. 2008, 46, 241-288. [CrossRef]

2. Cowan, J.J.; Sneden, C.; Lawler, J.E.; Aprahamian, A.; Wiescher, M.; Langanke, K.; Martínez-Pinedo, G.; Thielemann, F.K. Origin of the heaviest elements: The rapid neutron-capture process. Rev. Mod. Phys. 2021, 93, 015002. [CrossRef]

3. Kasen, D.; Badnell, N.R.; Barnes, J. Opacities and Spectra of the R-Process Ejecta From Neutron Star Mergers. Astrophys. J. 2013, 774, 25. [CrossRef]

4. Tanaka, M.; Kato, D.; Gaigalas, G.; Rynkun, P.; Radžiūtè, L.; Wanajo, S.; Sekiguchi, Y.; Nakamura, N.; Tanuma, H.; Murakami, I.; et al. Properties of Kilonovae from Dynamical and Post-merger Ejecta of Neutron Star Mergers. Astrophys. J. 2018, 852, 109. [CrossRef]

5. Kramida, A.; Ralchenko, Y.; Reader, J.; NIST ASD Team. Tex.Howpublished: NIST Atomic Spectra Database (ver. 5.8), National Institute of Standards and Technology, Gaithersburg, MD. 2020. Available online: https:/ / physics.nist.gov / asd (accessed on 14 June 2021). 
6. Meggers, W.F.; Corliss, C.H.; Scribner, B.F. Tables of Spectral-Line Intensities, Part I-Arranged by Elements; Number 145 in National Bureau of Standards Monograph; U.S. Government Printing Office: Washington, DC, USA, 1975.

7. Musiol, K.; Labuz, S. Experimental Determination of the Transition Probabilities in Er II. Phys. Scr. 1983, 27, 422-424. [CrossRef]

8. Bentzen, S.M.; Nielsen, U. Lifetime measurements in singly ionized erbium using fast-beam laser-modulation spectroscopy. J. Opt. Soc. Am. 1982, 72, 1210. [CrossRef]

9. Xu, H.; Jiang, Z.; Zhang, Z.; Dai, Z.; Svanberg, S.; Quinet, P.; Bi mont, E. Radiative lifetime measurements in Er II by time-resolved laser spectroscopy. J. Phys. B At. Mol. Opt. Phys. 2003, 36, 1771-1781. [CrossRef]

10. Lawler, J.E.; Sneden, C.; Cowan, J.J.; Wyart, J.F.; Ivans, I.I.; Sobeck, J.S.; Stockett, M.H.; Den Hartog, E.A. Improved Laboratory Transition Probabilities for Er II and Application to the Erbium Abundances of the Sun and Five r-Process-Rich, Metal-Poor Stars. Astrophys. J. Suppl. Ser. 2008, 178, 71-88. [CrossRef]

11. Stockett, M.H.; Den Hartog, E.A.; Lawler, J.E. Radiative lifetimes for 80 levels of singly ionized erbium. J. Phys. B At. Mol. Opt. Phys. 2007, 40, 4529-4536. [CrossRef]

12. Yu, Q.; Wang, X.; Li, Q.; Li, Y.; Dai, Z. Experimental Radiative Lifetimes, Branching Fractions, and Oscillator Strengths of Some Levels in Er i and Er ii. Astrophys. J. Suppl. Ser. 2019, 240, 25. [CrossRef]

13. Wyart, J.F.; Lawler, J.E. Theoretical interpretation and new energy levels in Er II. Phys. Scr. 2009, 79, 045301. [CrossRef]

14. Cowan, R. The Theory of Atomic Structure and Spectra; Los Alamos Series in Basic and Applied Sciences; University of California Press: Berkeley/Los Angeles, CA, USA, 1981.

15. Keraba Ankush, B. Experimental Verification of Theoretical Configuration Mixing in the Energy Levels of Er II Spectra via Isotope Shift Measurements Using a FTS. Am. J. Astron. Astrophys. 2017, 5, 10. [CrossRef]

16. Pedarnig, J.D.; Trautner, S.; Grünberger, S.; Giannakaris, N.; Eschlböck-Fuchs, S.; Hofstadler, J. Review of Element Analysis of Industrial Materials by In-Line Laser-Induced Breakdown Spectroscopy (LIBS). Appl. Sci. 2021, 11, 9274. [CrossRef]

17. Zhang, Y.; Zhang, T.; Li, H. Application of laser-induced breakdown spectroscopy (LIBS) in environmental monitoring. Spectrochim. Acta Part B At. Spectrosc. 2021, 181, 106218. [CrossRef]

18. Gonçalves, D.A.; Senesi, G.S.; Nicolodelli, G. Laser-Induced Breakdown Spectroscopy applied to environmental systems and their potential contaminants. An overview of advances achieved in the last few years. Trends Environ. Anal. Chem. 2021, 30, e00121. [CrossRef]

19. Fabre, C. Advances in Laser-Induced Breakdown Spectroscopy analysis for geology: A critical review. Spectrochim. Acta Part B At. Spectrosc. 2020, 166, 105799. [CrossRef]

20. Wiens, R.C.; Maurice, S.; Robinson, S.H.; Nelson, A.E.; Cais, P.; Bernardi, P.; Newell, R.T.; Clegg, S.; Sharma, S.K.; Storms, S.; et al. The SuperCam Instrument Suite on the NASA Mars 2020 Rover: Body Unit and Combined System Tests. Space Sci. Rev. 2021, 217, 4. [CrossRef] [PubMed]

21. Messaoud Aberkane, S.; Safi, A.; Botto, A.; Campanella, B.; Legnaioli, S.; Poggialini, F.; Raneri, S.; Rezaei, F.; Palleschi, V. Laser-Induced Breakdown Spectroscopy for Determination of Spectral Fundamental Parameters. Appl. Sci. 2020, $10,4973$. [CrossRef]

22. Putnam, R. Recent Advances in the Measurement of Rare-Earth Metal Transition Probabilities Using Laser-Induced Plasmas. Ph.D. thesis, University of Windsor, Windsor, ON, Canada, 2014.

23. Di Rocco, H.; Iriarte, D.; Pomarico, J. Lifetimes and transition probabilities of Xe II: Experimental measurements and theoretical calculations. Eur. Phys. J. D 2000, 10, 19-26. [CrossRef]

24. Mayo, R.; Ortiz, M.; Parente, F.; Santos, J.P. Experimental and theoretical transition probabilities for lines arising from the 6p configurations of Au II. J. Phys. B At. Mol. Opt. Phys. 2007, 40, 4651-4660. [CrossRef] 